DPNU-96-61

hep-th/9701036

December 1996

\title{
Dynamical Symmetry Breaking in Fractal Space
}

\author{
Yukinori Nagatani 6 \\ Department of Physics, Nagoya University, Nagoya 464-01, Japan
}

\begin{abstract}
We formulate field theories in fractal space and show the phase diagrams of the coupling versus the fractal dimension for the dynamical symmetry breaking. We first consider the 4-dimensional Gross-Neveu (GN) model in the $(4-d)$ dimensional randomized Cantor space where the fermions are restricted to a fractal space by the high potential barrier of Cantor fractal shape. By the statistical treatment of this potential, we obtain an effective action depending on the fractal dimension. Solving the $1 / N$ leading Schwinger-Dyson (SD) equation, we get the phase diagram of dynamical symmetry breaking with a critical line similar to that of the $d$-dimensional $(2<d \leq 4)$ GN model except for the system-size dependence. We also consider $\mathrm{QED}_{4}$ with only the fermions formally compactified to $d$ dimensions. Solving the ladder SD equation, we obtain the phase diagram of dynamical chiral symmetry breaking with a linear critical line, which is consistent with the known results for $d=4$ (the Maskawa-Nakajima case) and $d=2$ (the case with the external magnetic field).
\end{abstract}

\footnotetext{
${ }^{1}$ E-mail: nagatani@eken.phys.nagoya-u.ac.jp
} 


\section{Introduction}

In nature, we often see the fractals [1] in the shape of rivers or coastline [2], the shape of the electric sparks [3], the crystal growth and so on [4]. Then we are interested in what happens in the field theories when a fractal structure appears as a background structure.

In the quantum gravity the fractal-shaped space-time often appears [5], and we may expect that the quantum field theory in the fractal space-time can be regarded as a low energy effective theory of the quantum gravity.

In this paper, we first give a formulation of the 4-dimensional Gross-Neveu (GN) model [6] [7] in the randomized Cantor space as a sub-space of the 4dimensional space-time. (See Figure $\mathbb{1}-(\mathrm{b})(\mathrm{c})$ as an example of 2-dimensional

randomized Cantor space.) This randomized Cantor space is a fractal space which has the fractal dimension $4-d_{\text {Def }}$, where $d_{\text {Def }}$ means the deficit dimension. The $\left(4-d_{\text {Def }}\right)$-dimensional fractal subspace has no volume in 4 dimensions, then we take fractal renormalization generations $G$ to be large. We have two formulations of randomization of Cantor space; the type I randomization and the type II randomization. In this paper, we use the type II randomization mainly because of simplicity of the 2-point function. We discuss some feature of the type I in Appendix.

In this formulation, we compactify the 4-dimensional fermion to the type II randomized Cantor space by a high potential barrier, namely Cantor potential. The Cantor space has many holes as space-time deficits, then we put the high potential barrier to exclude the fermions from these holes. We adopt a cut-off level potential as the potential barrier. In the statistical sense of this randomness we can define the $n$-point function of the Cantor potentials, and we show the exact 1 and 2-point functions versus the deficit dimension $d_{\text {Def }}$ and the fractal generations $G$, where the 2-point function depends on the space-time configuration $(x, y)$ only through the integer-distance $g(x, y)$. (The typical 2-point function is described in Figure 2.) By means of a shift of the Cantor potential and the local correlation approximation of the 2-point function, this 2-point function generates the 4- 
fermion coupling related to the deficit dimensions.

By using the result of the $1 / N$ leading Schwinger-Dyson (SD) equation, we show the phase diagram of the dynamical symmetry breaking of the GN model in the fractal space (see Figure 1 ). This diagram is similar to that of the GN model in $d$ dimensions [9], but the critical line is curved rather than linear. This phase diagram depends on the system size; as the system size becomes larger, the symmetric region in the phase diagram gets smaller. There exists a critical dimension $4-d_{\text {Def }}^{*}$ depending on the system size. In other words, when we fix the fractal dimension, there exists a critical energy scale such that the theory in high energy is in the symmetric phase, while the system in the low energy is in the broken one.

Next we consider a generalization of the dynamics of fermion in the external constant magnetic field in $\mathrm{QED}_{4}$ [10] where the dynamical breaking of the chiral symmetry in weak coupling was shown [12]. In this theory, under the background magnetic field the fermion in the lowest Landau level has no freedom perpendicular to the magnetic field, and hence it is compactified to the $(1+1)$-dimensional magnetic tube. This dimensional reduction from 4 dimensions to 2 dimensions is the reason for the chiral symmetry breaking at any coupling $\alpha>0$ [12].

We then give in the last section a formal generalization of the 2-dimensional compactification of charged fermions by the external magnetic fields [12] to the $d$ dimensional fermion compactification. We apply this formulation to the $\mathrm{QED}_{4}$, in which the photon has 4-dimensional degree of freedom but the charged fermions have only $d$-dimensional one.

By the ladder SD equation [11], we show the phase diagram of the dynamical chiral symmetry breaking (See Figure 5). This phase diagram has a linear critical line which is very similar to the $d$-dimensional GN model [9]. We can understand that this phase diagram is a kind of analytic continuation between the 4-dimensional case without dimensional reduction [11] and the case of the fermion compactified into 2 dimensions by the external magnetic field [12]. 


\section{Cantor Spaces}

We can make the ordinary 2-dimensional Cantor Space by the renormalization sequence as follows (See Figure 1ㅏ-(a)). Suppose that there is a square space namely "parent-square". At the first step, we make a 1/3-scale square hole in the center of this space, by which there appear $(9-1)$ "sub-square" surrounding the center hole. At the second step, we zoom up to triple scale, by which there appear 8 square spaces, and we regard them as parent-squares of the next generation. For any square spaces, we return to the first step.

We call this 2 steps sequence as 1 generation. The exact Cantor space is generated by infinity-generations sequence. In any scale, this has a fractal dimension

$$
d_{\text {Fractal }}=\frac{\log \left(3^{2}-1\right)}{\log 3}=1.89 \cdots
$$

which is slightly smaller than 2 dimensions.

Now, we can generalize the parents-space dimensions from 2 to $D$ and the triple scale to $N$-multiple scale $(N, D \in \mathrm{Z})$, which we call $D$-dimensional $N$ Cantor space. This have the fractal dimension

$$
\begin{aligned}
d_{\text {Fractal }} & =\frac{\log \left(N^{D}-1\right)}{\log N}=D-d_{\text {Def }}, \\
d_{\text {Def }} & \simeq \frac{1}{N^{D} \log N},
\end{aligned}
$$

where we defined the deficit dimension $d_{\text {Def }}$ which means the effect of holes as the deficit of space.

But this exact D-dimensional $N$-Cantor space has no $D$-dimensional volume measure; Volume $=0$. Then we take finite generations $G$ to be large, and we have a finite volume:

$$
\begin{aligned}
\text { Volume } & =1-\left[\frac{1}{N^{D}}+\frac{1}{N^{D}}\left(\frac{N^{D}-1}{N^{D}}\right)+\cdots+\frac{1}{N^{D}}\left(\frac{N^{D}-1}{N^{D}}\right)^{G-1}\right] \\
& =\left(1-\frac{1}{N^{D}}\right)^{G} \simeq \exp -\frac{G}{N^{D}} .
\end{aligned}
$$

This $N$-Cantor space consists of only one shape, because there is a square hole in the center of any square space in any generation. 
However, we can select a position of hole of the same size at random from $N^{D}$ positions of sub-space rather than in the center of parent-square. Then we can extend this $N$-Cantor space to randomized ones as a class of spaces which have the same fractal dimensions (See Figure 1 1 -(b)(c)). It is the advantage of this randomized $N$-Cantor spaces class that we can treat the spaces statistically.

We note that we have two types of randomization. The type I randomization is to randomize the only position of holes and fix the number of holes. The type II randomization is to put holes with probability $p=1 / N^{D}$ per 1 subsquare independently so that any parent-square has one hole as average. Each randomization has the same fractal dimension as the ordinary Cantor space. We adopt the type II randomization for simplicity of the 2-point function. We will discuss the type I randomized Cantor space in Appendix.

When we consider the field theory in this Cantor space, we may have two formulations. The first one is to treat these holes in space as a boundary condition of fields. When the space-time dimension $D$ is equal to one, this formulation is easier but is very difficult technically in higher dimensional space like 4 dimensions. The second one is to treat these holes as high potential barriers to exclude fermions from these holes. We put potential

$$
V(x)= \begin{cases}0 & (x \in \text { ordinary space }) \\ \Lambda & (x \in \text { holes })\end{cases}
$$

where $\Lambda$ means the cut-off energy of the theory. We call $V(x)$ the Cantor potential.

For $D$-dimensional randomized $N$-Cantor potential class which is the potential version of randomized $N$-Cantor space, we can define the $n$-point function as the statistical meaning of randomness

$$
\begin{aligned}
\langle V(x)\rangle_{N(d)} & :=\int_{N(d): \mathrm{fix}} \mathcal{D} V V(x), \\
\langle V(x) V(y)\rangle_{N(d)} & :=\int_{N(d): \mathrm{fix}} \mathcal{D} V V(x) V(y),
\end{aligned}
$$

with normalization $\int_{N(d) \text { :fix }} \mathcal{D} V=1$. 
We can calculate the 1-point function easily as the average of the co-space volume of $N$-Cantor space:

$$
\begin{aligned}
\frac{1}{\Lambda}\langle V(x)\rangle_{N(d)} & =p+(1-p) p+\cdots+(1-p)^{G-1} p \\
& =1-(1-p)^{G}=1-\left(1-\frac{1}{N^{D}}\right)^{G}
\end{aligned}
$$

where we have defined

$$
p=\frac{1}{N^{D}}
$$

as a probability of the existence of hole per sub-square.

\section{2-Point Function}

To get the 2-point function of the $N$-Cantor space class, we use renormalization analysis. Let the 1-generation be the finest or high-energy one at the cut-off scale $\Lambda$ and the $G$-generation be the system-size one.

We can represent any point $x$ in the $D$-dimensional $G$-generation $N$-Cantor space as the $G$-columns of $N^{D}$-digit numbers:

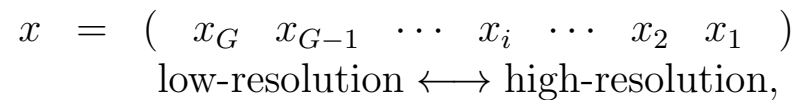

where $x_{i}$ has values from 1 to $N^{D}$ as a position of sub-square in $i$-generation; the point $x$ exists in the $x_{i}$-th sub-square.

In general, if we pick up any two points $x$ and $y$ in the $N$-Cantor space, we can regard them as the same point with respect to resolutions from the $G$-generation to some generation $g+1(0 \leq g \leq G)$, and as different points with respect to higher resolutions from $g$-generation to the finest generation 1 :

$$
\begin{aligned}
& x=\left(\begin{array}{lllllll}
x_{G} & x_{G-1} & \cdots & x_{g+1} & x_{g} & \cdots & x_{1}
\end{array}\right) \\
& y=\left(\begin{array}{ccccccc}
\| & \| & \cdots & \| & \mathbb{W} & \cdots & (\mathbb{W}) \\
y_{G} & y_{G-1} & \cdots & y_{g+1} & y_{g} & \cdots & y_{1}
\end{array}\right)
\end{aligned}
$$

In other words, for any two points $x$ and $y$, there exists a certain integer $g=$ $g(x, y) \geq 0$ such that it satisfies (10). We can prove that $g(x, y)$ satisfies

$$
g(x, x)=0
$$




$$
\begin{aligned}
& g(x, y)=g(y, x) \\
& g(x, z) \leq g(x, y)+g(y, z)
\end{aligned}
$$

namely $g(x, y)$ is a distance in the Cantor space. We call $g(x, y)$ a integer-distance.

When we fix the generations $G$, for any pairs of points $(x, y)$ such that $g(x, y)$ is a constant, we cannot distinguish pairs with respect to the statistical feature of pair. Then the 2-point function depends on the space-time configuration $(x, y)$ only through the integer-distance $g(x, y)$ :

$$
\frac{1}{\Lambda^{2}}\langle V(x) V(y)\rangle_{N, G}=L(g(x, y) ; G)_{N}
$$

This $L(g ; G)$ depends on the parents-space dimension $D$, integer $N$ which determines the fractal dimension of Cantor space, the generations of system $G$, and the integer-distance $g$ as the space-time dependence of 2-point function. $L(g ; G)$ is a decreasing function of $g$. We call $L(g ; G)$ a level, $L(G ; G)$ a base level and $L(0 ; G)$ a top level. The dependence of each levels on $D$ and $N$ is only through the probability $p$ in (8). The top level $L(0 ; G)$ is self-correlation, and hence

$$
\begin{aligned}
L(0 ; G)_{N, G} & =\frac{1}{\Lambda^{2}}\langle V(x) V(x)\rangle=\frac{1}{\Lambda}\langle V(x)\rangle \\
& =1-(1-p)^{G} .
\end{aligned}
$$

By the way, we can also get this result by renormalization transformation:

$$
\begin{aligned}
L(0 ; 1) & =p \\
L(0 ; 2) & =p+(1-p) L(0 ; 1), \\
& \vdots \\
L(0 ; G+1) & =p+(1-p) L(0 ; G) \\
& =\operatorname{Reno}[L(0 ; G)],
\end{aligned}
$$

where we defined

$$
\operatorname{Reno}[X]:=p+(1-p) X
$$

This "Reno" transformation is a renormalization transformation of probability from smaller generation to larger one. The first term of (15) is a probability of 
existence of larger hole and the second term is that of inheritance of the fine structure. The result of $n$-times of "Reno" transformations is

$$
\begin{aligned}
\operatorname{Reno}^{(n)}[X] & =1-(1-p)^{n}+(1-p)^{n} X \\
& =L(0 ; n)+(1-L(0 ; n))^{n} X .
\end{aligned}
$$

For the base level $L(G ; G)$, the distance between $x$ and $y$ is maximum with respect to $g(x, y)$, and there is no correlation between potential values $V(x)$ and $V(y)$. The base level is a probability that both $V(x)$ and $V(y)$ have non-zero value, then this level is only the square of these probabilities:

$$
L(G ; G)=(L(0 ; G))^{2}
$$

For the intermediate levels $L(g ; G) \quad(0 \leq g \leq G)$, in the way similar to the top levels we have

$$
\begin{aligned}
L(g ; g) & =(L(0 ; g))^{2}, \\
L(g ; g+1) & =p+(1-p) L(g ; g), \\
& \vdots \\
L(g ; G+1) & =p+(1-p) L(g ; G) \\
& =\operatorname{Reno}[L(g ; G)] .
\end{aligned}
$$

Then the intermediate levels are obtained from the base level by Reno transformations:

$$
\begin{aligned}
L(g ; G) & =\operatorname{Reno}^{(G-g)}[L(g ; g)] \\
& =L(G-g ; G)+(1-L(G-g ; G))(L(g ; g))^{2} \\
& =1-2(1-p)^{G}+(1-p)^{G+g} \\
& =1-2\left(1-\frac{1}{N^{D}}\right)^{G}+\left(1-\frac{1}{N^{D}}\right)^{G+g} .
\end{aligned}
$$

This result is consistent with both the top level $L(0 ; G)$ and the base level $L(G ; G)$. We show the typical resultant levels in Figure 2 when the parent-space dimension $D$ is 1 . 


\section{Local Correlation Approximation}

For our purpose, we shift the potential

$$
V(x) \longrightarrow \tilde{V}(x)=V(x)-\langle V(x)\rangle
$$

for $\langle\tilde{V}(x)\rangle=0$. Then the 2-point functions become

$$
\langle\tilde{V}(x) \tilde{V}(y)\rangle=\langle V(x) V(y)\rangle-\langle V(x)\rangle^{2} .
$$

The base level of $\langle V(x) V(y)\rangle$ is equal to $\langle V(x)\rangle^{2}$, then $\langle\tilde{V}(x) \tilde{V}(y)\rangle$ has no base level, and has only one peak in $x=y$ point. Then we approximate this 2-point function by the Dirac delta-function, namely "Local Correlation Approximation":

$$
\langle\tilde{V}(x) \tilde{V}(y)\rangle \simeq \frac{\Lambda^{2}}{\lambda^{D}} \Omega \delta^{(D)}(x-y)
$$

where $\lambda$ has a mass dimension which is a mass scale of the system size, and $\Omega$ means dimensionless volume of this peak (See Figure Q). $\Omega$ can be estimated through rewriting 2-point function of $\tilde{V}(x)$ :

$$
\langle\tilde{V}(x) \tilde{V}(y)\rangle=\Delta_{g(x, y)}^{G}+\Delta_{g(x, y)+1}^{G}+\cdots+\Delta_{G-1}^{G}
$$

where

$$
\begin{aligned}
\Delta_{g}^{G} & :=L(g ; G)-L(g+1 ; G) \\
& =p(1-p)^{G+g} \\
& =\frac{1}{N^{D}}\left(1-\frac{1}{N^{D}}\right)^{G+g}
\end{aligned}
$$

for $0 \leq g<G$. Then the volume of peak is

$$
\begin{aligned}
\Omega & =\sum_{g=0}^{G-1} \frac{N^{D g}}{N^{D G}} \Delta_{g}^{G} \\
& =\frac{1}{N^{D}} \frac{1}{N^{D}-2}\left(1-\frac{1}{N^{D}}\right)^{2 G}\left\{1-\left(\frac{1}{N^{D}-1}\right)^{G}\right\} \\
& \simeq \frac{1}{N^{2 D}}\left(1-\frac{1}{N^{D}}\right)^{2 G} .
\end{aligned}
$$




\section{Gross-Neveu Model in Fractal Space}

The action of the 4-dimensional Gross-Neveu model [7] is

$$
S[\psi]:=\int d^{4} x \bar{\psi} i \gamma^{\mu} \partial_{\mu} \psi+\frac{G_{0}}{2 N_{F}}(\bar{\psi} \psi)^{2},
$$

where $G_{0}$ is the bare coupling constant whose mass dimension is -2 and $N_{F}$ is the number of fermions. Through the $1 / N_{F}$ expansion analysis it is known that there is a critical coupling $G_{0}^{*}=4 \pi^{2} / \Lambda^{2}$. If $G_{0}<G_{0}^{*}$, then there is no dynamical mass of the fermion and the theory has an unbroken discrete chiral symmetry, whereas for $G_{0}>G_{0}^{*}$ the dynamical mass is generated and the theory is in a dynamically broken phase of the symmetry.

The action of the Gross-Neveu model with extra potential $\tilde{V}(x)$ is given by

$$
S[\psi, V]:=S[\psi]-\frac{1}{\sqrt{N_{F}}} \int d^{4} x V(x) \bar{\psi} \psi,
$$

where we omitted the tilde from $\tilde{V}$. We define the effective action of the GrossNeveu model with randomized $N$-Cantor potentials in Euclidean space:

$$
e^{-\tilde{S}_{\mathrm{eff}}[\psi]}:=\int \mathcal{D} V e^{-\tilde{S}[\psi, V]},
$$

where $\tilde{S}=$ Kinetic + Potential means Euclidean action (when Minkowski action is $S=$ Kinetic - Potential). To get the effective action we approximate this functional integral as:

$$
\begin{aligned}
& e^{- \text {Potential }}=1-\int d^{4} x\langle V(x)\rangle(\bar{\psi} \psi)(x) \\
&+\frac{1}{2} \int d^{4} x \int d^{4} y\langle V(x) V(y)\rangle(\bar{\psi} \psi)(x)(\bar{\psi} \psi)(y)+\cdots \\
& \simeq \exp \left[\frac{1}{2} \int d^{4} x \int d^{4} y\langle V(x) V(y)\rangle(\bar{\psi} \psi)(x)(\bar{\psi} \psi)(y)\right],
\end{aligned}
$$

where we used the definition of $n$-point function and absence of 1-point function, and we neglected more than 3-point functions as an approximation. Then the approximated effective action is

$$
S_{\mathrm{eff}}[\psi]:=\int d^{4} x \bar{\psi} i \gamma^{\mu} \partial_{\mu} \psi
$$




$$
\begin{aligned}
& +\int d^{4} x \int d^{4} y \frac{1}{2 N_{F}}\left[G_{0} \delta(x-y)+\langle V(x) V(y)\rangle\right] \\
& (\bar{\psi} \psi)(x)(\bar{\psi} \psi)(y) \\
& \simeq \int d^{4} x \bar{\psi} i \gamma^{\mu} \partial_{\mu} \psi+\frac{G_{\mathrm{eff}}}{2 N_{F}}(\bar{\psi} \psi)^{2}
\end{aligned}
$$

where we used local correlation approximation in the last equation and we defined effective 4-fermion coupling constant

$$
G_{\mathrm{eff}}=G_{0}+\frac{\Lambda^{2}}{\lambda^{4}} \Omega
$$

Our resultant effective action (30) takes the same form as the one of the original GN model, and the coupling constant is shifted as the effect of the fractal potential.

Let us define the dimensionless 4-fermion coupling:

$$
\begin{aligned}
g_{0} & :=\frac{\Lambda^{2} G_{0}}{4 \pi^{2}} \\
g_{\mathrm{eff}} & :=\frac{\Lambda^{2} G_{\mathrm{eff}}}{4 \pi^{2}}
\end{aligned}
$$

then the effective coupling reads

$$
g_{\mathrm{eff}}=g_{0}+\frac{1}{4 \pi^{2}}\left(\frac{\Lambda}{\lambda}\right)^{4} \Omega
$$

and the critical coupling in 4 dimensions becomes $g_{0}^{*}=1$. The equation of the critical line of the dynamical symmetry breaking becomes

$$
g_{0}+\frac{1}{4 \pi^{2}}\left(\frac{\Lambda}{\lambda}\right)^{4} \Omega=1
$$

Now we can discuss the phase diagram in fractal dimension $4-d_{\text {Def }}$ versus bare coupling $g_{0}$. By using

$$
\begin{aligned}
\Omega & =\frac{1}{N^{8}}\left(1-\frac{1}{N^{4}}\right)^{2(G-1)}, \\
d_{\text {Def }} & =\frac{1}{N^{4} \log N}, \\
\frac{\Lambda}{\lambda} & =N^{G},
\end{aligned}
$$


we further rewrite the equation of critical line (33) into

$$
g_{0}+\frac{d_{\text {Def }}^{2}\left(\log N\left(d_{\text {Def }}\right)\right)^{2}}{4 \pi^{2}}\left(\frac{\Lambda}{\lambda}\right)^{4-2 d_{\text {Def }}}=1
$$

which gives the phase diagram shown in Figure 4 . This phase diagram depends on the cut-off scale ratio $\Lambda / \lambda$. If we fix the ultraviolet cut-off $\Lambda$, then the phase depends on the system scale $\lambda$. When system size gets larger, the symmetric phase region in the phase diagram gets smaller.

There exists a critical dimension $4-d_{\text {Def }}^{*}$ which depends on the scale ratio $\lambda / \Lambda$. When we approximate $\log N\left(d_{\text {Def }}\right)$ to a constant $c$, we get a critical deficit dimension:

$$
d_{\mathrm{Def}}^{*} \simeq \frac{2 \pi}{c}(\lambda / \Lambda)^{2}
$$

If we fix the fractal dimension, then there is a kind of critical scale:

$$
\lambda^{*} \simeq \Lambda \sqrt{\frac{d_{\operatorname{Def}} c}{2 \pi}}
$$

above which (in the high energy) the system is in symmetric phase, while in the low energy it is in the broken phase.

\section{Strong $\mathrm{QED}_{4}$ with $d$-dimensional fermion}

In the $\mathrm{QED}_{4}$ with background constant magnetic field, it was found 12 that the dynamical mass of fermion $m_{d}$ is generated even in the weak coupling region, since the 4-dimensional fermion is compactified to a 2-dimensional one in the lowest Landau level (LLL):

$$
S\left(p_{/ /}, p_{\perp}\right)=i \frac{1}{p_{/ /}-m_{d}} e^{-l^{2} p_{\perp}^{2}} P_{\text {spin }},
$$

where $P_{\text {spin }}$ means the spin projection. This propagator has two directions; $p_{\perp}$ is a direction perpendicular to the constant magnetic field, while $p_{/ /}$is the rest which includes the time direction. For $p_{\perp}$ direction, the propagator is Gaussiandamping with length scale $l=1 / \sqrt{e B}$ which is the scale of the magnetic field. We call this direction a compact one. As for the $p_{\perp}$ direction, it is simply a free 
propagator with dynamical mass $m_{d}$ which will be determined by SD equation later. We call this direction a free one.

Then we will formally generalize this 2-dimensional compactification to $d$ dimensional one, and see whether or not the fermion mass generation takes place. We assume 4-dimensional momentum $p$ and its volume measure $d^{4} p$ can be decomposed into two-parts:

$$
\begin{aligned}
p= & p_{\text {free }}+p_{\text {comp }} \\
d^{4} p= & d^{d} p_{\text {free }} d^{4-d} p_{\text {comp }} \\
& p_{\text {free }} \perp p_{\text {comp }}
\end{aligned}
$$

where $p_{\text {free }}$ means the $d$-dimensional momentum like $p_{/ /}$, and $p_{\text {comp }}$ means the $(4-d)$-dimensional momentum like $p_{\perp}$. We call $p_{\text {free }}$ as a free direction and $p_{\text {comp }}$ a compactified one.

Based on the analogy with the constant magnetic case, we assume the fermion propagator as

$$
S\left(p_{\text {free }}, p_{\text {comp }}\right)=i \frac{1}{p_{\text {free }}-m_{d}} e^{-l^{2} p_{\text {comp }}^{2}}
$$

where $l$ means the compactified length like a magnetic scale $1 / \sqrt{e B}$. This propagator is very formal, but after formally contraction of spinors we can construct SD equation based on this. Thus our starting point is just the SD equation. In this propagator there are no spin projections, but in $d=2$ the SD equation coincides with that of the $\mathrm{QED}_{4}$ with background magnetic field [12].

In the case of the background magnetic field the photon propagator is the same as the 4-dimensional one without magnetic field. Then in our case we assume the same Landau-gauge photon propagator:

$$
D_{\mu \nu}(k)=\frac{i}{k^{2}}\left[g_{\mu \nu}-\frac{k_{\mu} k_{\nu}}{k^{2}}\right] .
$$

The Euclidean ladder SD equation in 4 dimensions with the bare mass equal to zero is

$$
m\left(p^{2}\right)=\frac{3 g^{2}}{(2 \pi)^{4}} \int_{0}^{\Lambda^{2}} d^{4} k \frac{m\left(k^{2}\right)}{k^{2}+m^{2}\left(k^{2}\right)} \frac{1}{(k-p)^{2}}
$$


By using (40) for $S\left(p_{\text {free }}, p_{\text {comp }}\right)$, we generalize (42) to the Euclidean ladder 4dimensional SD equation with $d$-dimensional fermion:

$$
\begin{aligned}
m\left(p_{\text {free }}^{2}\right)= & \frac{3 g^{2}}{(2 \pi)^{4}} \int_{0}^{\Lambda^{2}} d^{d} k_{\text {free }} \int_{0}^{\infty} d^{4-d} k_{\text {comp }} \\
& \frac{m\left(k_{\text {free }}^{2}\right)}{k_{\text {free }}^{2}+m^{2}\left(k_{\text {free }}^{2}\right)} e^{-l^{2} k_{\text {comp }}^{2}} \frac{1}{\left(k-p_{\text {free }}\right)^{2}},
\end{aligned}
$$

where

$$
\begin{aligned}
k & =k_{\text {free }}+k_{\text {comp }}, \\
k_{\text {free }} & \perp k_{\text {comp }}, \\
p_{\text {free }} & \perp k_{\text {comp }}, \\
k_{\text {free }} \cdot p_{\text {free }} & =\left|k_{\text {free }}\right|\left|p_{\text {free }}\right| \cos \theta .
\end{aligned}
$$

This $\theta$ means the angle between $k_{\text {free }}$ and $p_{\text {free }}$.

We can decompose these volume measures to radius and angular components:

$$
\begin{aligned}
m\left(p_{\text {free }}^{2}\right)= & \frac{1}{4 \sqrt{\pi}} \frac{\alpha}{\alpha_{c}} \frac{1}{\Gamma\left(\frac{d-1}{2}\right) \Gamma\left(\frac{4-d}{2}\right)} \\
& \int_{0}^{\Lambda^{2}} d k_{\text {free }}^{2} \int_{0}^{\infty} d k_{\text {comp }}^{2}\left(k_{\text {free }}^{2}\right)^{\frac{d-2}{2}}\left(k_{\text {comp }}^{2}\right)^{\frac{2-d}{2}} \frac{m\left(k_{\text {free }}^{2}\right)}{k_{\text {free }}^{2}+m^{2}\left(k_{\text {free }}^{2}\right)} e^{-l^{2} k_{\text {comp }}^{2}} \\
& \int_{0}^{\pi} d \theta \sin ^{d-2} \theta \frac{1}{k_{\text {free }}^{2}+k_{\text {comp }}^{2}+p_{\text {free }}^{2}-2\left|k_{\text {free }}\right|\left|p_{\text {free }}\right| \cos \theta},
\end{aligned}
$$

where we have defined:

$$
\frac{3 g^{2}}{(2 \pi)^{4}}=\frac{3 \alpha}{4 \pi^{3}}=\frac{1}{4 \pi^{2}} \frac{\alpha}{\alpha_{c}},
$$

with $\alpha_{c}=\pi / 3$ being the critical coupling of ordinary 4-dimensional QED [11]. We can do this angular integral as

$$
\begin{aligned}
m\left(p_{\text {free }}^{2}\right)= & \frac{1}{4} \frac{\alpha}{\alpha_{c}} \frac{1}{\Gamma\left(\frac{d}{2}\right) \Gamma\left(\frac{4-d}{2}\right)} \\
& \int_{0}^{\Lambda^{2}} d k_{\text {free }}^{2} \int_{0}^{\infty} d k_{\text {comp }}^{2}\left(k_{\text {free }}^{2}\right)^{\frac{d-2}{2}}\left(k_{\text {comp }}^{2}\right)^{\frac{2-d}{2}} \frac{m\left(k_{\text {free }}^{2}\right)}{k_{\text {free }}^{2}+m^{2}\left(k_{\text {free }}^{2}\right)} e^{-l^{2} k_{\text {comp }}^{2}} \\
& \frac{1}{k_{\text {free }}^{2}+k_{\text {comp }}^{2}+p_{\text {free }}^{2}} \mathrm{~F}\left[\frac{1}{2}, 1, \frac{d}{2} ; \frac{4 k_{\text {free }}^{2} p_{\text {free }}^{2}}{\left(k_{\text {free }}^{2}+k_{\text {comp }}^{2}+p_{\text {free }}^{2}\right)^{2}}\right],
\end{aligned}
$$


where $F$ means the hyper-geometric function.

We approximate $m\left(k_{\text {free }}^{2}\right)$ in the denominator of the fermion propagator as a constant dynamical mass $m_{d}$ à la Miransky [11]. Then this equation is the homogeneous linear integral equation:

$$
m\left(p_{\text {free }}^{2}\right)=\frac{\alpha}{4 \alpha_{c}} \int_{0}^{\Lambda^{2}} d k_{\text {free }}^{2} K\left(p_{\text {free }}^{2}, k_{\text {free }}^{2}\right) m\left(k_{\text {free }}^{2}\right) .
$$

In this equation, kernel $K$ is given by

$$
\begin{aligned}
K\left(p_{\text {free }}^{2}, k_{\text {free }}^{2}\right)= & \frac{1}{\Gamma\left(\frac{d}{2}\right) \Gamma\left(\frac{4-d}{2}\right)}\left(k_{\text {free }}^{2}\right)^{\frac{d-2}{2}} \frac{m\left(k_{\text {free }}^{2}\right)}{k_{\text {free }}^{2}+m_{d}^{2}} \tilde{K}\left(p_{\text {free }}^{2}, k_{\text {free }}^{2}\right), \\
\tilde{K}\left(p_{\text {free }}^{2}, k_{\text {free }}^{2}\right):= & \int_{0}^{\infty} d k_{\text {comp }}^{2} e^{-l^{2} k_{\text {comp }}^{2}}\left(k_{\text {comp }}^{2}\right)^{\frac{2-d}{2}} \\
& \frac{1}{k_{\text {free }}^{2}+k_{\text {comp }}^{2}+p_{\text {free }}^{2}} \mathrm{~F}\left[\frac{1}{2}, 1, \frac{d}{2} ; \frac{4 k_{\text {free }}^{2} p_{\text {free }}^{2}}{\left(k_{\text {free }}^{2}+k_{\text {comp }}^{2}+p_{\text {free }}^{2}\right)^{2}}\right],
\end{aligned}
$$

where we defined a symmetric sub-kernel $\tilde{K}\left(p_{\text {free }}^{2}, k_{\text {free }}^{2}\right)=\tilde{K}\left(k_{\text {free }}^{2}, p_{\text {free }}^{2}\right)$.

Since it is very difficult to solve this integral equation, we approximate this sub-kernel as follows. Note that in the 4-dimensional limit $(d \rightarrow 4)$, this subkernel becomes simpler:

$$
\lim _{d \rightarrow 4} \tilde{K}\left(p_{\text {free }}^{2}, k_{\text {free }}^{2}\right)=\frac{\theta\left(p_{\text {free }}^{2}-k_{\text {free }}^{2}\right)}{p_{\text {free }}^{2}}+\frac{\theta\left(k_{\text {free }}^{2}-p_{\text {free }}^{2}\right)}{k_{\text {free }}^{2}},
$$

corresponding to the ordinary 4-dimensional SD equation of $\mathrm{QED}_{4}$. Then we approximate this sub-kernel as

$$
\tilde{K}\left(p_{\text {free }}^{2}, k_{\text {free }}^{2}\right)=\theta\left(p_{\text {free }}^{2}-k_{\text {free }}^{2}\right)\left(p_{\text {free }}^{2}\right)^{\frac{2-d}{2}}+\theta\left(k_{\text {free }}^{2}-p_{\text {free }}^{2}\right)\left(k_{\text {free }}^{2}\right)^{\frac{2-d}{2}} .
$$

By numerical calculation, we can check that this approximation is good for $2<$ $d<4$, while it is exact for $d=4$. We note that this approximated sub-kernel has no dependence on the compactified scale $l$, and hence the cut-off $\Lambda$ is the only scale parameter. Thus this approximation is useful to get a phase diagram, although it may not for getting a concrete value of the dynamical mass.

By this approximation, we can translate this integral equation to hypergeometric differential equation:

$$
\frac{d}{d p_{\text {free }}^{2}}\left(\left(p_{\text {free }}^{2}\right)^{\frac{d}{2}} \frac{d}{d p_{\text {free }}^{2}} m\left(p_{\text {free }}^{2}\right)\right)+\frac{\alpha}{4 \alpha_{c}} \frac{d-2}{2} \frac{\left(p_{\text {free }}^{2}\right)^{\frac{d}{2}}}{p_{\text {free }}^{2}+m_{d}^{2}} m\left(p_{\text {free }}^{2}\right)=0
$$


for $\left(0 \leq p_{\text {free }}^{2} \leq \Lambda^{2}\right)$, with an IR boundary condition:

$$
\left[\left(p_{\text {free }}^{2}\right)^{\frac{d}{2}} \frac{d}{d p_{\text {free }}^{2}} m\left(p_{\text {free }}^{2}\right)\right]_{p_{\text {free }}^{2}=0}=0
$$

and a UV boundary condition at cut-off $\Lambda^{2}$ :

$$
\left[\left(p_{\text {free }}^{2} \frac{d}{d p_{\text {free }}^{2}}+\frac{d-2}{2}\right) m\left(p_{\text {free }}^{2}\right)\right]_{p_{\text {free }}^{2}=\Lambda^{2}}=0 .
$$

We get the solution satisfying the IR condition:

$$
\begin{aligned}
m\left(p_{\text {free }}^{2}\right) & =C F\left[a_{+}, a_{-}, \frac{d}{2} ;-\frac{p_{\text {free }}^{2}}{m_{d}^{2}}\right] \\
a_{ \pm} & =\frac{d-2}{4}\left(1 \pm \sqrt{1-\frac{\alpha}{\alpha_{c}} \frac{2}{d-2}}\right)
\end{aligned}
$$

where $C$ denotes a constant with a mass dimension containing $\Lambda$. (The exact solution would have $C$ also containing $l$.) This solution oscillates, when $a_{ \pm}$is imaginary, for

$$
\frac{d-2}{2}<\frac{\alpha}{\alpha_{c}}
$$

For $d=4$, it is well known [11] that the non-trivial solutions of SD equation exist only for the oscillating case. Then we can draw the phase diagram of dynamical breaking of the chiral symmetry (see Figure 5).

There is a linear critical line, which is very similar to that of the $d$-dimensional $(2<d<4)$ Gross-Neveu model or Nambu-Jona-Lasinio model, namely the models with naive dimensional reduction of space-time 9].

For $d=4$ and $d=2$, our result is consistent with the known results [11] [12].

\section{Conclusions and Discussions}

We have studied the 4-dimensional Gross-Neveu (GN) model [7] in the $(4-d)$ dimensional fractal space. We adopted the 4-dimensional type II randomized $N$-Cantor space (Figure $\mathbb{f}-(\mathrm{c})$ ) as a fractal space-time. The reason why we used the Cantor space is that this space is useful to control its fractal dimension. Its fractal dimension is $4-d_{\text {Def }}$. Cantor spaces have holes as space-time deficit. To 
consider fermions in the Cantor space, we treated fermions in the background cut-off level potential, namely, Cantor potential to exclude fermions from the holes. We adopted the randomized Cantor space, and then we were able to treat Cantor potential as statistical objects. There are two formulations of randomization of the Cantor space or potential; the type I randomization and the type II one. In the type I randomization, we randomized the only position of holes while preserving the number of holes. In Appendix, we discussed the 2-point function of the type I randomization, the effective action of the GN model in this randomized Cantor space and its difficulty in the $1 / N_{F}$ leading order. In the type II randomization, we put holes at random while preserving the average number of holes. We adopted the type II randomization because of simplicity of the 2-point function.

We defined $n$-point functions and we calculated 1- and 2-point functions for this potential exactly, where the 2-point function depends on the space-time configuration $(x, y)$ only through the integer-distance $g(x, y)$ ([10). (the result of the type II is (10)(12)(19) and one of the type $\mathrm{I}$ is (67)). By the local correlation approximation (22), we obtain the effective action (30) of the GN model in $\left(4-d_{\text {Def }}\right)$-dimensional fractal space. In our effective action, the contribution of the fractal effect appears as a shift of the 4-fermion coupling; when the fractal dimension becomes smaller (than 4-dimension), the 4-fermion coupling gets bigger.

As a result of the $1 / N$ leading SD equation [7], we got the phase diagram on the bare 4-fermion coupling versus fractal dimension of the space-time (Figure 4 ). In this phase diagram the critical line (35) depends on the system size through the ratio $\Lambda / \lambda$. This phase diagram implies that the 4-fermion coupling gets bigger as the fractal dimension becomes smaller than 4-dimension. There exists a critical dimension $d^{*}=4-\frac{2 \pi}{c}(\lambda / \Lambda)^{2}$, which depends on the system size. When we fix UV cut-off $\Lambda$ and fractal dimension, there is a kind of critical scale $\lambda^{*}$ (37) such that in high energy the system is in the symmetric phase, while in low energy it is in the broken phase. In the equation of the critical line (35) when $d \leq 2$, the scale dependence is inverted. Our analysis is valid only for small 
deficit dimension, although we are also interested in whether or not some critical phenomena happens in $d=2$. At this moment we do not know how to treat large deficit dimension.

There is some similarity between this analysis and the GN model in $d$ dimensions or with naive dimensional reduction [9]. But our phase diagram depends on the system size in contrast to the naive dimensional reduction. Hence the naive dimensional reduction analysis does not imply the fractal space analysis.

In the last section, we have taken another approach to $\mathrm{QED}_{4}$ with the fermions living in non-integer dimensional space-time. Based on the analysis of ref. [12], we studied $\mathrm{QED}_{4}$ with $d$-dimensional fermion and ordinary 4-dimensional photon. We treated fermion which has propagator with $d$-dimensional free direction and $(4-d)$-dimensional compactified direction (40). We have solved the ladder $\mathrm{SD}$ equation (47). Under a certain approximation to the kernel of the integral equation (51), we got the phase structure (see Figure 5). This phase diagram is consistent with the 4-dimensional ladder QED [11] and the same model in the external magnetic field with fermion dimensional reduction by 2 [12]. Our phase diagram is similar to that of the $d$-dimensional GN model with naive dimensional reduction [9].

We think we can also apply the randomized Cantor space analysis to $\mathrm{QED}_{4}$ by the analysis of gauged NJL [13].

\section{Acknowledgments}

I would like to thank Prof. K. Yamawaki for helpful suggestions and discussions, and also for careful reading the manuscript. I also appreciate helpful suggestions of H. Katou and T. Ito. I am indebted to the Japan Society for the Promotion of Science (JSPS) for its financial support as a JSPS Junior Fellow. The work is

supported in part by a Grant-in-Aid for Scientific Research from the Ministry of Education, Science and Culture. 


\section{A The Type I Randomization of Cantor Space}

In this appendix, we discuss the $D$-dimensional type I randomized $N$-Cantor potential.

The type I randomization is to randomize the only position of holes and fix the number of holes to one per parent-square. In the type II randomization, the number of holes per parent-square is not constant, and only the average of number is equal to 1 . We show the 2-dimensional 3-Cantor spaces; the ordinary space, the type I and the type II randomized space in Figure 1 .

The 1-point function of both randomization is the same:

$$
\begin{aligned}
\frac{1}{\Lambda}\langle V(x)\rangle_{N(d)} & =\frac{1}{N^{D}}+\frac{1}{N^{D}}\left(\frac{N^{D}-1}{N^{D}}\right)+\cdots+\frac{1}{N^{D}}\left(\frac{N^{D}-1}{N^{D}}\right)^{G-1} \\
& =1-\left(1-\frac{1}{N^{D}}\right)^{G} .
\end{aligned}
$$

We can also get this result by renormalization transformation:

$$
\begin{aligned}
L(0 ; G+1) & =\frac{1}{N^{D}}+\frac{N^{D}-1}{N^{D}} L(0 ; G) \\
& =\operatorname{Reno}[L(0 ; G)]
\end{aligned}
$$

where we defined the renormalization transformation

$$
\operatorname{Reno}[X]:=\frac{1}{N^{D}}+\frac{N^{D}-1}{N^{D}} X
$$

like (15). The result of $n$-times of these transformations is

$$
\operatorname{Reno}^{(n)}[X]:=L(0 ; n)+\left(\frac{N^{D}-1}{N^{D}}\right)^{n} X .
$$

The 2-point function is slightly different from type II randomization, but the outline and the top level are the same as the type II. The 2-point function depends only on the integer-distance $g(x, y)$ as a space-time dependence:

$$
\frac{1}{\Lambda^{2}}\langle V(x) V(y)\rangle_{N, G}=L(g(x, y) ; G)_{N},
$$

where $L(g ; G)$ is an increasing function of $g$. This nature is the same as the type II. The top level $L(G ; G)$ is a self-correlation, then

$$
\begin{aligned}
L(G ; G)_{N, G} & =\frac{1}{\Lambda^{2}}\langle V(x) V(x)\rangle=\frac{1}{\Lambda}\langle V(x)\rangle \\
& =1-\left(1-\frac{1}{N^{D}}\right)^{G} .
\end{aligned}
$$


The top level is the same as that of the type II.

The difference from type II appears in the base level and intermediate levels. For the base level $L(G ; G)$, in the way similar to 1-point function we have

$$
\begin{aligned}
L(1 ; 1) & =0 \\
L(2 ; 2) & =\frac{2}{N^{D}} L(0 ; 1)+\frac{N^{D}-2}{N^{D}}[L(0 ; 1)]^{2}, \\
& \vdots \\
L(G+1 ; G+1) & =\frac{2}{N^{D}} L(0 ; G)+\frac{N^{D}-2}{N^{D}}[L(0 ; G)]^{2},
\end{aligned}
$$

where $2 / N^{D}$ means a probability of $x \in$ hole or $y \in$ hole, and this hole means the biggest one in any generation. Then we get the result:

$$
L(G ; G)=1-2\left(\frac{N^{D}-1}{N^{D}}\right)^{G}+\left(\frac{N^{D}-2}{N^{D}}\right)\left(\frac{N^{D}-1}{N^{D}}\right)^{2(G-1)} .
$$

There is a renormalization relation among the intermediate levels $L(g ; G)$ for $0 \leq g \leq G$ :

$$
L(g ; G+1)=\operatorname{Reno}[L(g ; G)]
$$

where we remember the "Reno" transformation creates a larger structure preserving the fine structures. This relation is the same as the type II. Then the intermediate levels $L(g ; G) \quad(0 \leq g<G)$ are obtained from the base level $L(g ; g)$ by $(G-g)$-times Reno transformation:

$$
\begin{aligned}
L(g ; G) & =\operatorname{Reno}^{(G-g)}[L(g ; g)] \\
& =1-2\left(\frac{N^{D}-1}{N^{D}}\right)^{G}+\left(\frac{N^{D}-2}{N^{D}}\right)\left(\frac{N^{D}-1}{N^{D}}\right)^{G+g-2} .
\end{aligned}
$$

Finally, we have resultant levels:

$$
L(g ; G)= \begin{cases}1-2\left(\frac{N^{D}-1}{N^{D}}\right)^{G}+\left(\frac{N^{D}-2}{N^{D}}\right)\left(\frac{N^{D}-1}{N^{D}}\right)^{G+g-2} & (0<g \leq G), \\ 1-\left(\frac{N^{D}-1}{N^{D}}\right)^{G} & (g=0)\end{cases}
$$

(see Figure [3). 
We note $L(G ; G)<L(0 ; G)^{2}$, with the difference between them being

$$
\begin{aligned}
\Delta & :=L(G ; G)-L(0 ; G)^{2} \\
& =-\frac{1}{N^{2 D}}\left(\frac{N^{D}-1}{N^{D}}\right)^{2(G-1)} .
\end{aligned}
$$

This is the most important difference from the type II randomization.

When we shift the potential

$$
V(x) \longrightarrow \tilde{V}(x)=V(x)-\langle V(x)\rangle
$$

for $\langle\tilde{V}(x)\rangle=0$, the 2-point function becomes

$$
\langle\tilde{V}(x) \tilde{V}(y)\rangle=\langle V(x) V(y)\rangle-\langle V(x)\rangle^{2}
$$

Since the base level of $\langle V(x) V(y)\rangle$ is close to $\langle V(x)\rangle^{2},\langle\tilde{V}(x) \tilde{V}(y)\rangle$ has small negative base level $\Delta$ and has only one peak in the $x=y$ point.

By the "Local Correlation Approximation", 2-point function becomes

$$
\langle\tilde{V}(x) \tilde{V}(y)\rangle \simeq \frac{\Lambda^{2}}{\lambda^{D}}\left[\lambda^{D} \Delta+\Omega \delta^{(D)}(x-y)\right],
$$

where $\lambda$ has a mass dimension which is a mass scale of the system size, and $\Omega$ means dimensionless volume of this peak.

We rewrite 2-point function to get $\Omega$ :

$$
\langle\tilde{V}(x) \tilde{V}(y)\rangle=\Delta_{g(x, y)}^{G}+\Delta_{g(x, y)+1}^{G}+\cdots+\Delta_{G-1}^{G}+\Delta
$$

where

$$
\begin{aligned}
\Delta_{g}^{G} & :=L(g ; G)-L(g+1 ; G) \\
& = \begin{cases}\frac{1}{N^{D}}\left(\frac{N^{D}-2}{N^{D}}\right)\left(\frac{N^{D}-1}{N^{D}}\right)^{G+g-2} & (1 \leq g<G), \\
\frac{1}{N^{D}}\left(\frac{N^{D}-1}{N^{D}}\right) & (g-1\end{cases}
\end{aligned}
$$

Then the volume of peak is

$$
\Omega=\sum_{g=0}^{G-1} \frac{N^{D g}}{N^{D G}} \Delta_{g}^{G}=\frac{1}{N^{2 D}}\left(\frac{N^{D}-1}{N^{D}}\right)^{2(G-1)} .
$$


This result is very similar to the one of the type II randomization. But note that $\Omega=-\Delta$, then the total volume of 2 -point function $\Omega+\Delta$ equals to zero.

The effective action of the 4-dimensional GN model in this Cantor potential becomes

$$
\begin{aligned}
S_{\mathrm{eff}}[\psi]:= & \int d^{4} x \bar{\psi} i \gamma^{\mu} \partial_{\mu} \psi \\
+ & \int d^{4} x \int d^{4} y \frac{1}{2 N_{F}}\left[\left(G_{0}+\frac{\Lambda^{2}}{\lambda^{4}} \Omega\right) \delta(x-y)-\Lambda^{2} \Omega\right] \\
& (\bar{\psi} \psi)(x)(\bar{\psi} \psi)(y) .
\end{aligned}
$$

Then the 4-fermion coupling in momentum space becomes

$$
G(k)=\left(G_{0}+\frac{\Lambda^{2}}{\lambda^{4}} \Omega\right)-(2 \pi)^{4} \Lambda^{2} \Omega \tilde{\delta}(k) .
$$

Because of a finite space-time volume characterized by the mass scale $\lambda$, this delta function $\delta \tilde{(k)}$ at $k=0$ stands for a finite value:

$$
(2 \pi)^{4} \tilde{\delta}(k)=\int d x e^{i k x}=\left\{\begin{array}{cc}
0 & (k \neq 0) \\
1 / \lambda^{4} & (k=0) .
\end{array}\right.
$$

The $1 / N_{F}$ leading $\mathrm{SD}$ equation now reads

$$
\begin{aligned}
i S(p)-\not p & =\frac{C_{G_{0}+\Lambda^{2} / \lambda^{4} \Omega}}{2} \\
& =G_{0} \int \frac{d^{4} k}{(2 \pi)^{4}} \operatorname{tr} S(k),
\end{aligned}
$$

where the solid lines mean fermion line which takes the form

$$
S^{-1}(p)=i\left(B\left(p^{2}\right)-A\left(p^{2}\right) p\right)
$$

and the dotted lines mean the constant non-local 4-fermion interaction. Then the SD equation (78) is the same as the one of the ordinary 4-dimensional GN model.

In the type I randomized Cantor space, we conclude that the $1 / N_{F}$ leading $\mathrm{SD}$ equation cannot describe the dependence on the fractal dimension. We must analyze next to the leading order of $1 / N_{F}$ expansion. 


\section{References}

[1] B.B. Mandelbrot, Fractal-From, Chance and Dimension (Freeman, Sanfrancisco, 1977); The Fractal Geometry of Nature (Freeman, Sanfrancisco, 1982); H. Takayasu, Fractal (Asakura, Tokyo, 1987).

[2] B.B. Mandelbrot, Science 156, (1967) 636; T. Takagi Science of Form (Kohdansha, Tokyo, 1984).

[3] L. Niemeyer, L. Pietronero and H. J. Wiesmann, Phys. Rev. Lett. 52, (1984) 1033.

[4] T.A. Witten and L.M. Sander, Phys. Rev. Lett. 47, (1981) 1400; M. Matsushita, M. Sano, Y. Hayakawa, H. Honjo and Y. Sawada, Phys. Rev. Lett. 53, (1984) 286; M. Matsushita, Y. Hayakawa, and Y. Sawada, Phys. Rev. A32, (1985) 3814.

[5] H. Kawai, N. Kawamoto, T. Mogami, and Y. Watabiki, Phys. Lett. B306, (1993) 19; H. Aoki, H. Kawai, J. Nishimura and A. Tsuchiya, Nucl. Phys. B474, (1996) 512.

[6] Y. Nambu and G. Jona-Lasinio, Phys. Rev. 122, (1961) 345.

[7] D. Gross and A. Neveu, Phys. Rev. D10, (1974) 3235; E. Witten, Nucl. Phys. B145 (1978) 110.

[8] H.J. Munczek, Phys. Lett. B175, (1986) 215; D. Atkinson, H.J. de Groot and P.W. Johnson, Phys. Rev. D43, (1991) 218.

[9] K. Kikukawa and K. Yamawaki, Phys. Lett. B234, (1990) 497; K. Kondo, M. Tanabashi and K Yamawaki, Prog. Theor. Phys. 89, (1993) 1249.

[10] J. Schwinger, Phys. Rev. 82, (1951) 664; Phys. Rev. D7, (1973) 1696.

[11] T. Maskawa and H. Nakajima, Prog. Theor. Phys. 52, (1974) 1326; Prog. Theor. Phys. 54, (1975) 860; R. Fukuda and T. Kugo, Nucl. Phys. B117, (1976) 250; V.A. Miransky, Il. Nuovo Cim. 90A, (1985) 149. 
[12] V.P. Gusynin, V.A. Miransky, and I.A. Shovkovy, Phys. Rev. Lett. 73, (1994) 3499; Phys. Rev. D52, (1995) 4718; Phys. Lett. B349, (1995) 477; Phys. Rev. D52, (1995) 4747; Nucl. Phys. B462, (1996) 249.

[13] W.A. Bardeen, C.N. Leung and S.T. Love, Phys. Rev. Lett. 56 (1986) 1230; K. Kondo, H. Mino and K. Yamawaki Phys. Rev. D39, (1989) 2430; T. Appelquist, M. Soldate, T. Takeuchi and L.C.R. Wijewardhana, in Proc. Johns Hopkins Workshop on Current Problems in Particle Theory 12, Baltimore, 1988, eds. G. Domokos and S. Koresi-Domokos (World Scientific, Singapore, 1988); K. Kondo, M. Tanabashi and K Yamawaki, in ref. [9]. 


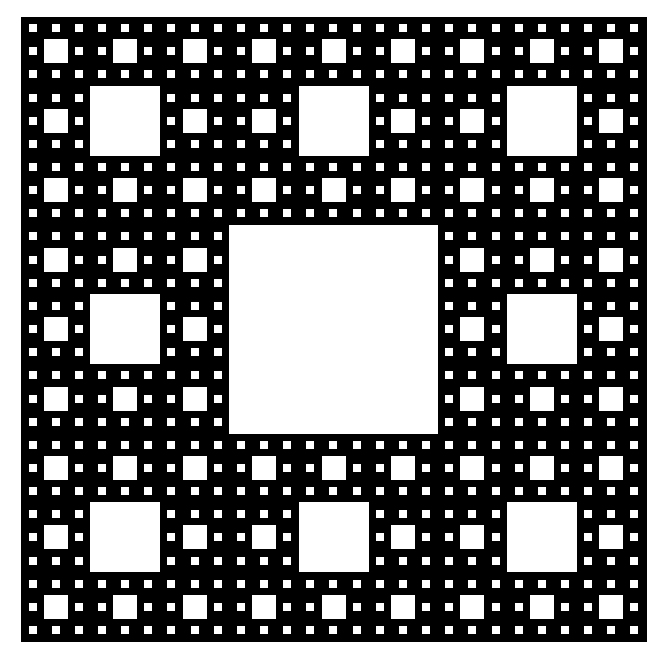

(a) 3-Cantor space

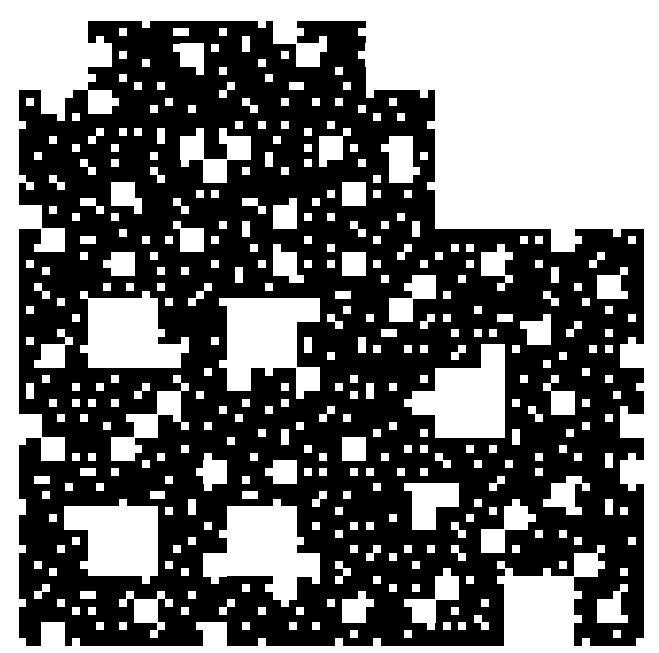

(b) Type I randomized 3-Cantor space

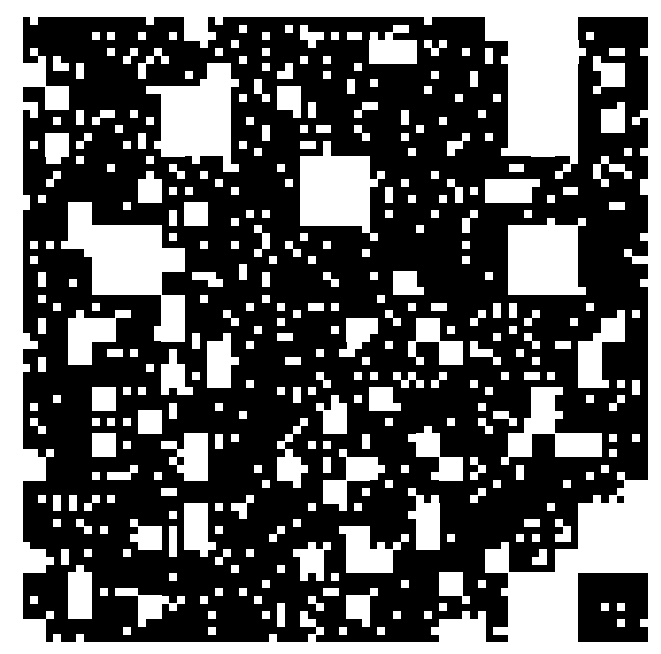

(c) Type II randomized 3-Cantor space

Figure 1: 2-dimensional 3-Cantor spaces. (a)Ordinary 3-Cantor space. Black part means Cantor space and white part means holes. Any square space has a hole at the center position. (b)Type I randomized 3-Cantor space. The difference from (a) is only the selection of the hole position out of 9 positions. (c)Type II randomized 3-Cantor space. The number of holes is not constant. The average number of holes is equal to (a) and (b). Every space has the same fractal dimension $d=\log 8 / \log 3=1.89 \cdots$. 


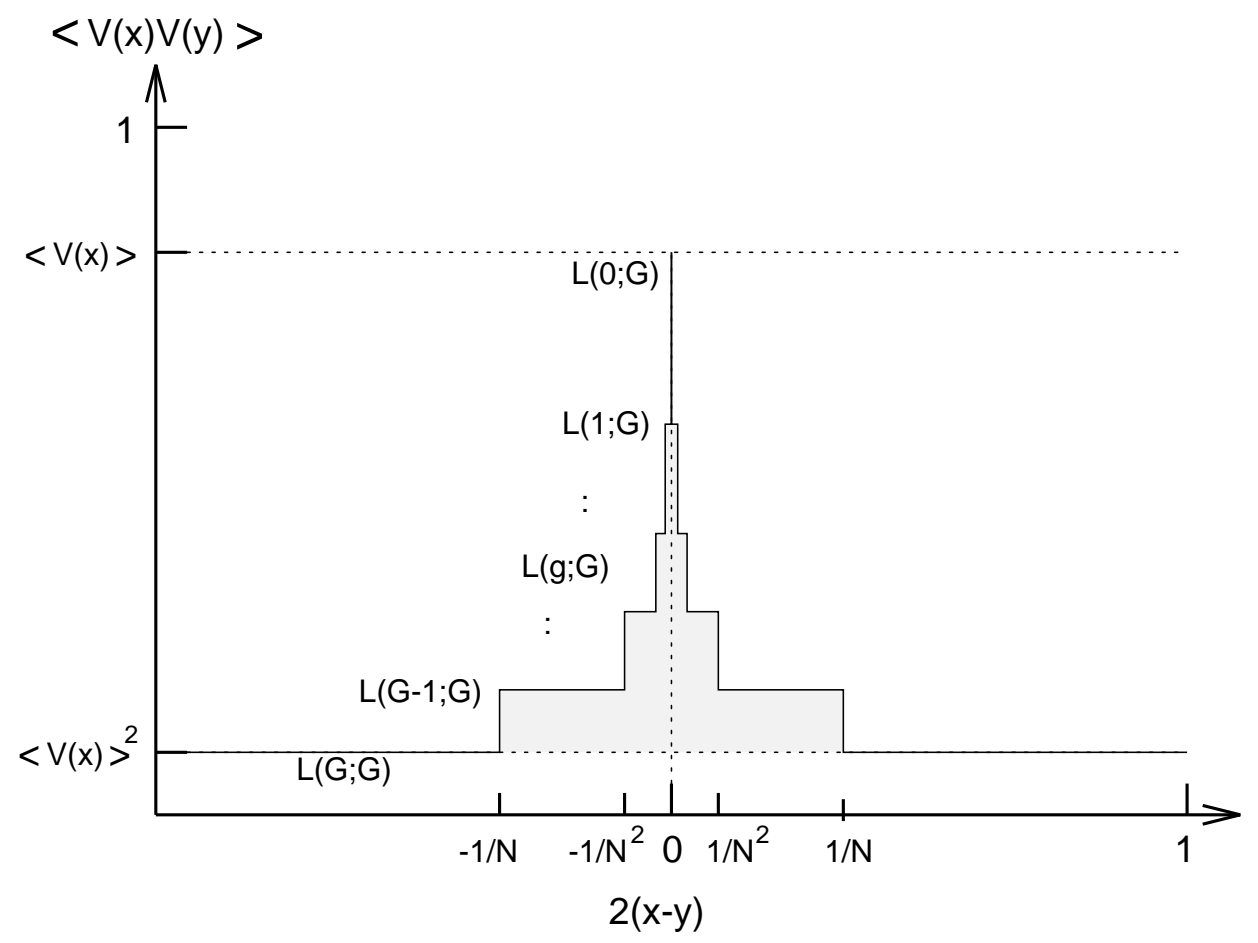

Figure 2: Typical 2-point correlation function of the 1-dimensional type II randomized $N$-Cantor potential. The exact form depends on both $x$ and $y$ through the integer-distance $g(x, y)$. For $D$-dimensional case, we get the 2-point correlation function by replacing $N$ by $N^{D}$ in $L(G ; g)$. In the local potential approximation, we define the weight of delta function $\Omega$ as the volume of the shadow zone. 


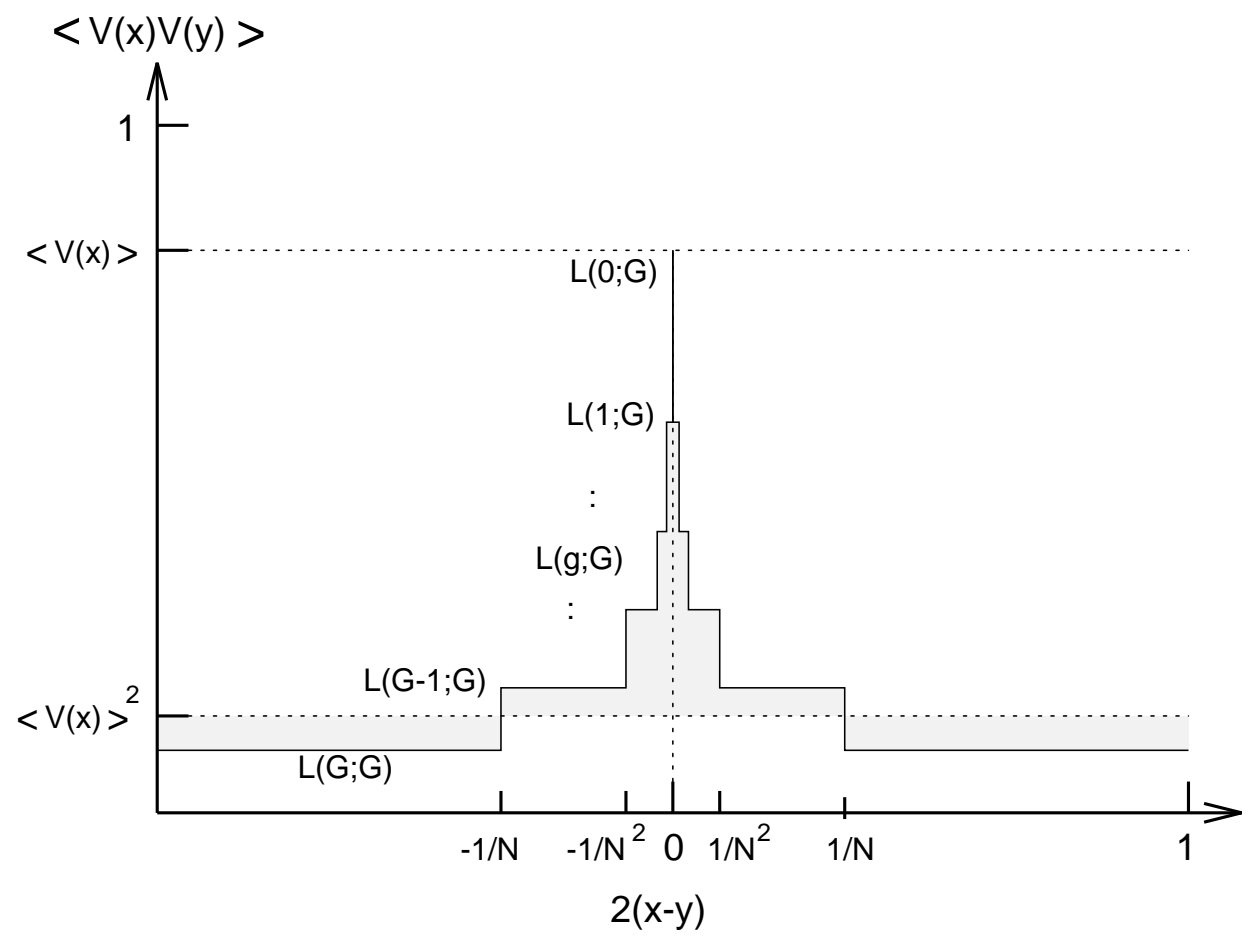

Figure 3: Type I typical 2-point correlation function of the 1-dimensional randomized $N$-Cantor potential. There are non-local parts. (See Appendix.) 


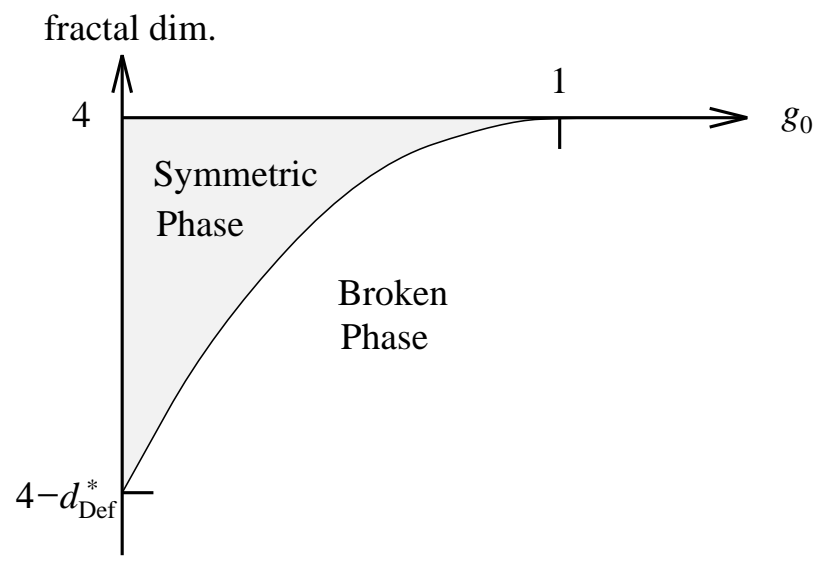

Figure 4: Phase diagram of the Gross-Neveu model in the 4-dimensional $N(d)$-Cantor space which has fractal dimension $\left(4-d_{\text {Def }}\right)$. In this diagram $g_{0}$ is the ordinary dimensionless 4 -fermion bare coupling, and $4-d_{\text {Def }}^{*}$ means the critical fractal coupling. This phase diagram depends on $\Lambda / \lambda$ where $\Lambda$ is the cut-off of 4 -fermion theory and $\lambda$ is the scale of the system size. The critical deficit dimension depends on the system size like $d_{\text {Def }}^{*} \simeq \frac{2 \pi}{c}(\lambda / \Lambda)^{2}$.

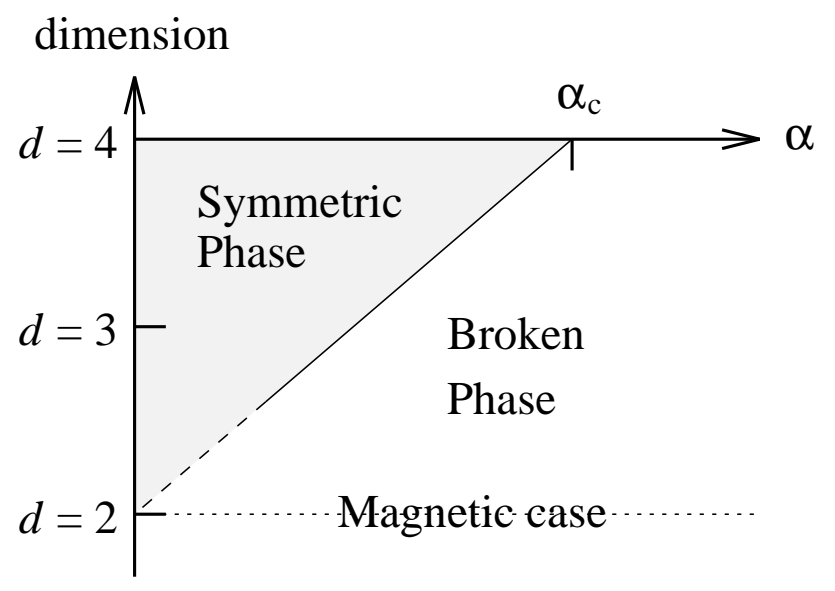

Figure 5: Phase diagram of the chiral symmetry breaking in $\mathrm{QED}_{4}$ with $d$-dimensional fermion. This is the diagram of QED coupling $\alpha$ versus free fermion dimension $d . \alpha_{c}=\frac{\pi}{3}$ means the critical coupling of $\mathrm{QED}_{4}$. The $d=4$ line corresponds to the strong $\mathrm{QED}_{4}$ phase diagram. The $d=2$ line corresponds to one of the $\mathrm{QED}_{4}$ with the external constant magnetic field. 